\title{
ASTM Standards in the SEM
}

\author{
John J. Friel*
}

*Princeton Gamma-Tech, C/N 863, Princeton, NJ 08542

The goal of image analysis in microscopy is the quantification of the microstructure. To this end, there are many ASTM standards that specify procedures in microscopy, and several of them are either specific to electron microscopes or apply to both electron- and light-optical microscopes. A brief description of some of these is listed in Table 1.

The standards are available in the ASTM Annual Book of Standards [1], and those listed in Table 1 can be found in Volume 03.01. They are also available on CD-ROM for keyword searching. While there are other standards that relate to the microscopy of specific classes of materials, such as steel or paper, the ones reviewed here all relate to some aspect of quantitative microscopy or microanalysis. They are all under the jurisdiction of ASTM Committee E04 on Metallography.

E 562 provides the statistical basis for point counting a discrete phase. It supplies guidance on sample selection and preparation, and it provides tables to determine the required number of points and fields to achieve a given level of precision. The standard also includes measures of precision and bias based on interlaboratory round-robin testing. Although it was written for manual measurement, automatic image analysis (AIA) can be used, and the measurements can be made directly on a microscope or photomicrograph.

E 766 is a standard practice for calibrating the magnification of an SEM. During its last revision in 1998, it was broadened to permit the use of any magnification calibration standard. The procedure describes calibration in both the $\mathrm{x}$ - and $\mathrm{y}$-directions as well as calibration of the scale marker. A table is provided for recording the data.

E 986 is a standard practice for SEM beam size characterization. It describes an experiment for measuring the electron beam diameter with suitable precautions to achieve reproducible results. During its recent revision in 2002, the standard was updated to incorporate digital microscopes.

E 1245 describes stereological measurements and quantification of the amount and distribution of inclusions or second phase particles in a matrix. It describes sample selection, preparation, and system calibration. The method is designed for AIA, and it reports: $\mathrm{N}_{\mathrm{A}}, \mathrm{N}_{\mathrm{L}}, \mathrm{A}_{\mathrm{A}}$, average area, average length, and mean free path. The 95\% confidence interval and \%relative accuracy are also calculated. This standard is applicable to many types of microstructures, and it produces results of known precision.

E 1268 is a method for assessing the degree of banding or orientation within a microstructure. It requires measurements to be made on two perpendicular axes and reports an anisotropy index (AI) and a measure called $\Omega_{12}$. It also contains photomicrographs of various microstructures to illustrate the applicability of the method. 
E 1508 is a standard guide to quantitative analysis by EDS. Although the guide is not intended to replace a textbook on microanalysis, it suggests good operating practice. Parameters that are under control of the analyst are identified, and guidance on their use is provided. The standard also reports precision and bias based on an interlaboratory round-robin testing program, using both standards and standardless methods.

E 2142 is a standard that was published in 2002 after several years of development. This standard calls for the use of SEM/EDS to analyze inclusions in steel, but it is applicable to other discrete phases as well. As steelmaking practice produces steels with fewer inclusions, the LOM is not always adequate to characterize their size and shape, and certainly not their composition. In this case, the SEM is used for its higher resolution, and EDS provides the chemical composition. In the most commonly used inclusion rating standard (E 45), the chemistry of inclusions is inferred from their shape or reflectivity. Now E 2142 makes a direct determination of composition and provides for either a traditional classification or a user-defined one. The traditional classification of inclusions is: types A, B, C, and D, which are inferred to be sulfides, aluminates, silicates, and globular oxides respectively, but in E 2142, the automated chemical classification program of the EDS computer is set up to suit the application. Complex or duplex inclusions analyzed by this method tend to be resolved into their components, especially at higher magnifications.

TABLE 1. Designation and brief description of some ASTM standards for use in the SEM.

\begin{tabular}{lll}
\hline ASTM Designation & Applicability & Brief description \\
\hline E 562 & LOM/SEM & Point counting \\
E 766 & SEM & Magnification calibration \\
E 986 & SEM & Beam diameter measurement \\
E 1245 & LOM/SEM & Stereological measurement of discrete phases \\
E 1268 & LOM/SEM & Degree of banding \\
E 1508 & SEM & Guide to quantitative analysis by EDS \\
E 2142 & SEM & Classifying inclusions by SEM/EDS \\
\hline
\end{tabular}

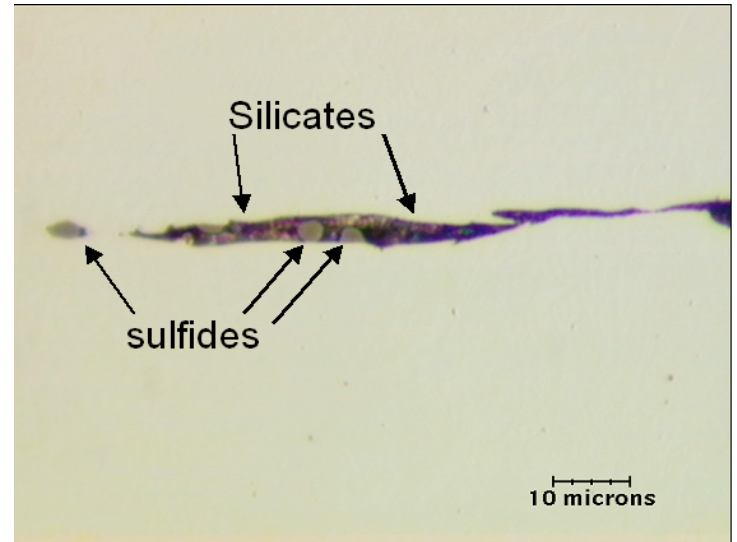

FIG. 1. Duplex silicate/sulfide inclusion
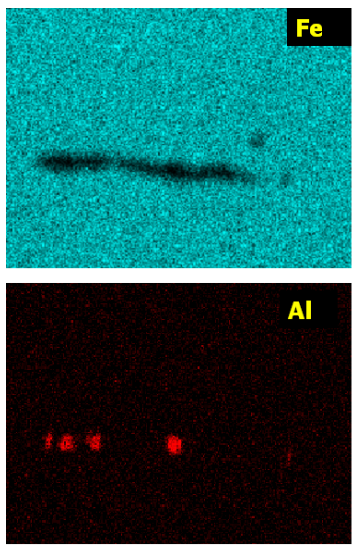

FIG. 2. X-ray maps of duplex aluminate/sulfide inclusion

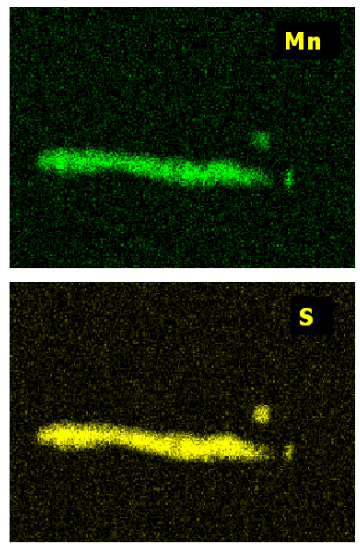

Mn

References

[1] ASTM Annual Book of Standards. 